\title{
Cardiorenal Syndrome Caused by Heart Failure with Preserved Ejection Fraction
}

\author{
Chiara Lazzeri, ${ }^{1}$ Serafina Valente, ${ }^{1}$ Roberto Tarquini, ${ }^{2}$ and Gian Franco Gensini ${ }^{1}$ \\ ${ }^{1}$ Heart and Vessel Department, Azienda Ospedaliero-Universitaria Careggi, 50134 Florence, Italy \\ ${ }^{2}$ Department of Internal Medicine, University of Florence, 50134 Florence, Italy \\ Correspondence should be addressed to Chiara Lazzeri, lazzeric@libero.it
}

Received 25 August 2010; Accepted 3 January 2011

Academic Editor: Claudio Ronco

Copyright (c) 2011 Chiara Lazzeri et al. This is an open access article distributed under the Creative Commons Attribution License, which permits unrestricted use, distribution, and reproduction in any medium, provided the original work is properly cited.

\begin{abstract}
Since cardiorenal dysfunction is usually secondary to multiple factors acting in concert (and not only reduced cardiac output) in the present paper we are going to focus on the interrelationship between heart failure with normal ejection fraction and the development of cardiorenal syndrome. The coexistence of renal impairment in heart failure with preserved ejection fraction (CRS type 2 and 4) is common especially in older females with hypertension and/or diabetes. It can be hypothesized that the incidence of this disease association is growing, while clinical trials enrolling these patients are still lacking. The main mechanisms thought to be involved in the pathophysiology of this condition are represented by the increase of intra-abdominal and central venous pressure and the activation of the renin-angiotensin system. Differently from CRS in heart failure with reduced ejection fraction, the involvement of the kidney may be under-diagnosed in patients with heart failure and preserved ejection fraction and the optimal therapeutic strategy in this condition, though challenging, is far to be completely elucidated. Further studies are needed to assess the best therapeutic regimen in patients with renal dysfunction (and worsening) and heart failure and preserved ejection fraction.
\end{abstract}

\section{Introduction}

The cardiorenal syndrome (CRS) is a complex disease in which heart and kidney are simultaneously affected and their deleterious effects are reinforced in a feedback cycle, with accelerated progression of renal and myocardial damage [1-3].

The incidence of heart failure in the United States approaches 10 per 1000 in those older than 65 years and accounts for 1 million hospitalizations and 3 million office visits annually [4]. During the natural history of cardiac dysfunction, the critical importance of the cardiorenal interaction is emphasized by the fact that decreased renal function predicts cardiovascular mortality and complicates heart failure [5]. Baseline glomerular filtration rate (GFR) appears to be a stronger predictor of mortality in patients with HF than left ventricular ejection fraction or NYHA functional class [6]. In the Candesartan in Heart Failure: Assessment of Reduction in Mortality and Morbidity (CHARM), it was observed that impaired renal function was independently associated with heightened risk for death, cardiovascular death, and hospitalization for heart failure in patients with heart failure with both preserved as well as reduced LVEF [7]. Patients with chronic renal insufficiency are at strikingly higher risk for myocardial infarction, HF with systolic dysfunction, HF with preserved left ventricular ejection fraction, and death resulting from cardiac causes compared with individuals with normal GFR [8]. Conversely, reversal of renal dysfunction can improve cardiac function [9]. Hypertensive heart disease and HF with a normal ejection fraction are common among individuals with advanced and end-stage renal disease [10]. Renal disease patients with left ventricular hypertrophy have accelerated rates of coronary events and markers of uremia compared with those with normal left ventricular mass, and a high proportion of these individuals develop clinical HF [11]. It has recently been observed [12] that the $50 \%$ of patients with preclinical diastolic dysfunction had renal insufficiency that was defined by calculated creation clearance of $<60 \mathrm{~mL} / \mathrm{min}$. In these patients, proposed mechanisms for the progression of 
diastolic dysfunction include not only left ventricle stiffness, but also vascular stiffening (systemic and pulmonary) and volume expansion.

A more comprehensive characterization of the cardiorenal syndrome implicates the pathophysiologic disequilibrium between the heart and the kidney, in which malfunction of one organ consequently promotes the impairment of the other. Risk factors for its development include diabetes mellitus, hypertension, and a history of congestive heart failure or chronic renal failure.

Since cardiorenal dysfunction is usually secondary to multiple factors acting in concert (and not only reduced cardiac output) in the present paper we are going to focus on the interrelationship between heart failure with normal ejection fraction and the development of cardiorenal syndrome.

Firstly, we are going to summarize the recent evidence on heart failure with normal ejection fraction and on the cardiorenal syndromes. Then the main pathophysiologic mechanisms characterizing the cardiorenal syndrome developing in patients with heart failure and preserved ejection fraction (HFPEF) are hypothesized and the challenges in diagnosis and management of patients with cardiorenal syndrome and HFPEF are discussed.

\section{The Cardiorenal Syndrome}

The term cardiorenal syndrome (CRS) has been variably defined in the last decades without a well-accepted definition. Some investigators have suggested that this term should be used to describe patients with coexisting severe cardiac and renal dysfunction [13], while, more recently, a working group of the US National Heart, Lung, and Blood Institute used this term to describe the state in which advanced congestive heart failure (CHF) becomes complicated by acute impairment of kidney function [1-3]. According to these investigators, renal responses are thought largely to be the result of primary changes in cardiac function and CRS could be therefore defined as "a state in which therapy to relieve CHF symptoms is limited by further worsening renal function."

On the other hand, and from a broader point of view, taking into account the dynamic and close interplay between heart and kidney, the CRS has been recently viewed as "a pathophysiologic disorder of the heart and kidneys whereby acute or chronic dysfunction in one organ may induce acute or chronic dysfunction in the other organ" $[1-3,14,15]$.

As recently reported in the consensus conference of the Acute Dialysis Quality Initiative [3], the cardiorenal syndromes (CRS) were defined as "disorders of the heart and kidney whereby acute or chronic dysfunction in one organ may induce acute or chronic dysfunction of the other" and five different types of cardiorenal syndrome are described [1-3].

According to this classification, the worsening of renal function occurring in patients with heart failure and preserved ejection fraction may belong to CRS type 2. In this syndrome, chronic heart disease and CKD frequently coexist and it can be hardly distinguished which disease came first. In other words, most often CRS type 2 cannot be distinguished from CRS type 4, in which, among chronic heart disease the following conditions should be considered: cardiomyopathy, LV remodelling and dysfunction, diabetic cardiomyopathy [16] congenital heart disease, but also diastolic dysfunction.

\section{Heart Failure with Preserved Ejection Fraction}

The incidence of heart failure with preserved ejection fraction (HFPEF) is reported to include about $50 \%$ of the general heart failure population [17], while the prevalence of HFPEF is still increasing over the last years when compared to the prevalence of heart failure with reduced ejection fraction (HFREF) [18]. Its prevalence is higher in the elderly $[19,20]$ (especially in females); in a recent study of HFPEF, all patients were aged $>80 \mathrm{yrs}$, with a mean age of 87 [21].

Older age, hypertension, diabetes, obesity, and coronary artery disease are risk factors for both HFPEF and HFREF. [22]. In HFNEF, hypertension is a more common risk factor. While in HFREF ischemic heart disease is the most common etiology. In decompensated heart failure, $63 \%$ of patients with systolic and $54 \%$ of patients with diastolic heart failure have coronary artery disease [23].

As a result of modern evidence-based heart failure (HF) therapy, the prognosis of patients with heart failure with reduced left ventricular ejection fraction (HFREF) improved progressively over the past 3 decades. Conversely, despite frequent use of similar pharmacological agents, the prognosis of patients with heart failure with normal left ventricular ejection fraction (HFPEF) remained unaltered over the same time period [24-27].

It has been reported that the risk of sudden cardiac death is better correlated to left ventricular mass than to the ejection fraction [28]. The left ventricular mass is increased considerably in both HFPEF and HFREF; thus, the risk assessment for sudden cardiac death based on ejection fraction alone may not be appropriate [29].

Impaired left ventricular relaxation and increased passive stiffness is the principal functional derangement in HFPEF [30]. The pressure-volume relation during diastole shifts upward and to the left; as a result there is a disproportionately greater increase in diastolic pressure for any increase in volume. In HFPEF, because of the disproportionate increase in left ventricular diastolic pressure, there is an increase in left atrial and pulmonary venous pressure that is associated with symptoms and signs of pulmonary venous congestion [31]. Postcapillary pulmonary hypertension resulting from increased pulmonary venous pressure may precipitate right heart failure. Left ventricular stroke volume and cardiac output may also decline because of decreased end-diastolic volume (preload dependent). Currently, left diastolic but also nondiastolic abnormalities are discussed as possible reasons for HFPEF. Nondiastolic abnormalities may include an impairment of ventricular-vascular coupling [32], systolic left ventricular (LV) dyssynchrony, systolic and diastolic ventricular interactions (e.g., due to pericardial diseases, pulmonary hypertension), or chronotropic incompetence [33] 
as possible contributors to the heart failure symptomatology of these patients, while pathologies inducing changes in chamber compliance lead to diastolic abnormalities of the left ventricle [32].

\subsection{Main Mechanisms for the Pathophysiology of CRS Type 2 Associated with Heart Failure and Normal Ejection Fraction (Table 1)}

3.1.1. Intra-Abdominal and Central Venous Pressure Elevation. The Poiseuille law summarizes the relationship between blood pressure, cardiac output, and systemic vascular resistance. Cardiac flow is dependent on a sufficient pressure gradient across the body's capillary networks. HF (and HF with preserved left ejection fraction) is marked by an elevation in central venous pressure, which attenuates the gradient across the glomerular capillary network. Indeed, there is increasing evidence to support roles for elevated renal venous pressure and intraabdominal pressure (IAP) in the development of progressive renal dysfunction in patients with HF. In one early experiment, Winton [34] reported that urine formation by isolated canine kidney was markedly reduced at renal venous pressures of $20 \mathrm{mmHg}$ and abolished at pressures $>25 \mathrm{mmHg}$. Renal blood flow was also diminished in proportion to the decrease in pressure gradient across the afferent and efferent renal circulations, probably caused by the increased efferent arterial pressure. Rising renal venous pressure limited urine formation and renal blood flow more than a reduction in arterial pressure. Bradley and Bradley showed that abdominal compression to produce IAP of $20 \mathrm{mmHg}$ in normal individuals markedly reduced GFR and renal plasma flow. These relationships are supported by modern in vivo animal models [35]. In a broad spectrum of patients with cardiovascular disease, increased central venous pressure was associated with impaired renal function and independently associated with all-cause mortality. Interestingly the slope between CVP and impaired eGFR was steeper with relatively preserved cardiac function [36].

In patients who underwent elective cardiac surgery, preoperative presence of high CVP was a strong predictor of the occurrence of acute renal injury, independent of the presence of low cardiac output [37].

In the recent years, there has also been increasing recognition that oliguric acute renal dysfunction frequently accompanies abdominal compartment syndrome in surgical and trauma patients [38]. These changes are promptly reversed by abdominal decompression and may be associated with subsequent polyuria.

The concept that venous congestion, not arterial blood flow, is an important mediator of cardiorenal failure is supported by the findings of the Evaluation Study of Congestive Heart Failure and Pulmonary Artery Catheterization Effectiveness trial, in which only baseline right atrial pressure, not arterial blood flow, correlated with baseline serum creatinine [39].

Several mechanisms by which abdominal pressure might contribute to CRS have been explored. Elevation of renal
TABLE 1: Mechanisms thought to be involved in the pathogenesis of the cardiorenal syndrome in HFPEF patients.

\section{Main mechanisms}

(i) Intra-abdominal and central venous pressure elevation.

(ii) Activations of the renin-angiotensin systematic.

Other mechanisms

(i) Sympathetic overactivity.

(ii) Oxidative injury and endothelial dysfunction.

Precipitating factors

Disease conditions that is, Infections.

Drugs, that is, nonsteroidal inflammatory agents.

parenchymal pressure does not appear to have significant effects on GFR or renal blood flow. This was shown in studies of isolated porcine kidneys subjected to increasing amounts of extrinsic pressure [35]. Conversely, elevated central and renal venous pressures offer a stronger explanation for the relationship between elevated IAP and renal dysfunction. Elevating renal venous pressure by $30 \mathrm{mmHg}$ for 2 hours in intact porcine kidneys resulted in a substantial reduction in renal blood flow and GFR [35]. Furthermore, patients with HF with impaired renal function at baseline or worsening renal function during hospitalization have significantly elevated central venous pressure relative to those with less renal impairment [40]. In one study of intensive medical therapy directed at volume reduction, hemodynamic profiles were monitored in all patients with pulmonary artery catheters, and only elevated central venous pressure correlated with worsening versus preserved renal function [41]. The role of elevated central and renal venous pressures is further supported by the association of elevated jugular venous pulsations on physical examination with higher baseline serum creatinine and increased risk for hospitalization and death caused by pump failure [42]. Finally, the association of tricuspid regurgitation with renal dysfunction was recently examined in 196 consecutive patients with HF. The authors found that patients with at least moderate tricuspid regurgitation by transthoracic echocardiography had lower estimated GFR and that a linear relationship existed between severity of tricuspid regurgitation and degree of GFR impairment.

3.1.2. Renin-Angiotensin-Aldosterone Axis and Renal Dysfunction. The extreme sodium avidity and ventricular remodelling conferred by RAAS elaboration in HF are a maladaptive response to altered hemodynamics, sympathetic signaling, and progressive renal dysfunction.

On a therapeutic point of view, drugs that block the renin-angiotensin system reduce the progression of both heart and CKD. The optimal approach is the combination of ACE-I and beta-blocker, the titration of dosage. The addition of either an ARB or aldosterone antagonist is depending on clinical conditions and patients characteristics. Therapy of CHF and coexisting renal impairment is still not evidence-based, since these patients are often excluded from clinical trials. Since these patients are typically hypervolemic, 
more intensive diuretic therapy is needed. ACE-I and ARB initiation may cause deterioration in renal function, which is frequently transient and reversible. Anemia is often present in patients with CRS type 2 and correction of anemia may improve symptoms with no increase in survival [43].

Since the higher incidence of HF in the elderly, it should be remembered that these patients show increased susceptibility to renal dysfunction, impairment of sodium and water excretion, and postural hypotension, and aggravation of hypotension with the treatments (e.g., ACEinhibitors, b-blockers, nitrates, and hydralazine). Therapy has to be individualized and consider aging-specific changes in physiology, drug metabolism, drug pharmacokinetics and tolerance, comorbidities, polypharmacy, and drug-drug interactions.

\subsubsection{Other Mechanisms}

Sympathetic Overactivity. The adverse consequences of sympathetic nervous system activity to the heart are well known. Less well appreciated are the systemic effects of renal sympathetic stimulation. There are now good data to suggest that the renal sympathetic activation leads to direct vascular effects. A recent pilot study of catheter-based renal sympathetic denervation in patients with resistant hypertension found significant improvements in GFR in $24 \%$ of patients undergoing the procedure [43]. In an HF population, denervation could possibly affect renal function and halt renal sympathetic nerve-mediated progression of cardiac failure related to elaboration of catecholamines and the RAAS. Further investigation into this exciting concept is needed to determine whether it is clinically relevant.

3.1.4. Oxidative Injury and Endothelial Dysfunction. Neurohormones are strong precipitants and mediators of an oxidative injury cascade that leads to widespread endothelial dysfunction, inflammation, and cell death in the CRS. In this setting, AT-II seems to be particularly important, exerting many deleterious effects through the activation of NADPH oxidase and NADH oxidase. AT-II activates these 2 enzymes within vascular smooth muscle cells, cardiac myocytes, and renal tubular epithelial cells, generating superoxide, a reactive oxygen species [44-46]. Reactive oxygen species have many unfavourable effects in living tissues and likely contribute to the processes of aging, inflammation, and progressive organ dysfunction. Growing evidence supports oxidative injury as a common link between progressive cardiac and renal dysfunction. Because both primary cardiac failure and primary renal failure lead to elaboration of the RAAS, activation of oxidases by AT-II in one organ has the potential to lead to progressive dysfunction in the secondary organ through reactive oxygen species generation.

In summary, it can be hypothesized that, in patients with HFPEF and cardiorenal syndrome, congestion [40, 41] leading to a reduction in the arteriovenous pressure gradient across the kidney, as well as decrements in mean arterial pressure and renal perfusion pressure, tends to reduce glomerular filtration rate. Concomitantly, there is the pathophysiologic activation of the renin-angiotensinaldosterone system (RAAS) and the sympathetic nervous system (SNS) and the release of antidiuretic hormone, endothelin, cytokines, and various other inflammatory and vasoactive mediators that promote marked sodium and water retention, volume overload and adverse cardiovascular and renal remodeling. Under these conditions, another important mechanism contributing to cardiorenal dysfunction during the progression of heart failure is the deficiency in the production of compensatory natriuretic peptides and/or resistance to its renal actions $[47,48]$.

\subsection{Diagnostic and Therapeutic Interventions for Cardiorenal} Syndrome in HFPEF. So far there are no specific data on the diagnostic interventions in patients with cardiorenal syndrome and HFPEF.

Identifying the onset or progression of cardiorenal syndrome is paramount to proper management and can result in disease attenuation and prolonged survival both in patients with preserved EF and in those with reduced EF [49]. Though current research has been focusing on identifying markers that would permit an earlier or more accurate diagnosis of cardiorenal syndrome, no factor is specific for patients with HFPEF and CRS.

Neutrophil gelatinase-associated lipocalin (NGAL), a recently discovered acute kidney injury biomarker, indicating the accumulation of nephrotoxins and renal ischemia, typically precedes an elevation of creatinine by $48-72 \mathrm{~h}$. Cystatin $\mathrm{C}$, another acute kidney injury biomarker, has been suggested to be a better and earlier predictor of glomerular function than serum creatinine, as it is not affected by age, sex, race, or muscle mass.

B-type natriuretic peptide (BNP) or N-terminal proBNP (NT-pro-BNP) levels [50], which are general markers of $\mathrm{HF}$, can be evaluated when $\mathrm{HF}$ diagnosis is not certain. Plasma levels of BNP or Ntpro- BNP increase with left ventricular mass, wall stress, and filling pressures. To date, baseline levels of NT-pro-BNP of 339 and $409 \mathrm{pg} / \mathrm{mL}$ have been reported in patients with HF-PEF, higher than in normal subjects but less elevated than usually observed in decompensated HF with low EF. However the relationship between BNP, renal function, and the severity of heart failure is less clear [3], not only for diagnostic purposes, but also for the management of therapy [51].

In regard to the potential diagnostic role(s) of imaging techniques, there is no specific data for patients with CRS and HFNEF. In the future, non-invasive techniques (such renal vein blood flow assessment by Doppler technique) should be refined to quantify renal blood flow. These data could be then correlated with cardiac and renal biomarkers and to guide ongoing therapy.

Despite the importance of HFNEF, the treatment of this phenotype remains poorly understood [52]. Pharmacologic treatment of HFNEF patients is aimed to decrease blood pressure, promote regression of LV hypertrophy, prevent tachycardia, treat symptoms of congestion, and maintain atrial contraction as recommended by the ACC and the AHA joint guidelines [53]. Optimizing hemodynamics is primarily 
achieved through reduction of cardiac preload and afterload. ACE inhibitors and ARBs directly affect myocardial relaxation and compliance by blocking angiotensin II receptors, thereby reducing interstitial collagen deposition and fibrosis [54].

In many large, randomized, controlled clinical trials, researchers have assessed the beneficial effects of ACE inhibitors, b-block, and ARBs in HFREF patients, but these effects have not been established in HFNEF patients. Treatment recommendations are derived mainly from the large evidence-based trials that existed for management of HFREF [55] [Class I] or are based largely on the results of small, nonrandomized studies, clinical experience, and pathophysiologic reasoning [56] [Class III]. Recently, two large-scale HFNEF trials have reported their disappointing results: in the CHARMPreserved trial, the ARB candesartan produced a modest reduction in hospitalizations for $\mathrm{HF}$ but had no effect on mortality [57]; in PEP-CHF, the ACEinhibitor perindopril had similar effects.

No data are so far available specifically for patients with CRS and HFPEF. It has been recently observed that decreased eGFR was associated with an increased risk of early postmyocardial infarction (MI) HF, the association being strongest in patients with preserved ejection fraction, in whom it was an important independent predictor of HF. Though reninangiotensin-aldosterone blockade is well documented to reduce rates of late post-MI HF, particularly in patients with depressed EF, it is not known if intensive reninangiotensinaldosterone blockade during the acute phase of MI affects rates of early post-MI HF in patients with preserved EF and impaired renal function [58].

It can be speculated that the therapeutic targets in patients with CRS and HFPEF are represented mainly by the reduction of cardiac filling pressure while maintaining adequate volume status.

Restriction of sodium intake and the administration of diuretics may be beneficial through reduction of LV ventricular filling pressures. They are also useful in treating hypertension, which is a common trigger for worsening HFNEF. In the Hong Kong Diastolic Heart Failure, diuretics alone appeared to be effective in reducing symptoms and improving quality of life in HFNEF patients [59].

Diuretics should therefore be initiated at low dose and uptitrated gradually to achieve adequate urine output. Once volume status is normalized, maintenance often requires chronic oral diuretic therapy with active fluid restriction to forestall the tendency to volume overload created by sustained neurohormonal activation and enhanced thirst. Clinicians should bear in mind that if aggressive therapy is employed, diuretic-induced hypovolemia can result in severe renal injury or exacerbate any preexisting renal insufficiency.

Whenever diuretic resistance develops in these patients, treatment does not differ from that of patients with CRS and HFREF, including ultrafiltration, when needed $[3,5]$.

All the conditions able to aggravate renal injury (i.e., anemia and infections) should be timely identified and properly treated and, similarly medications able to adversely influence renal function (such as nonsteroidal inflammatory agents) should be interrupted.

\section{Conclusion}

The coexistence of renal impairment in heart failure with preserved ejection fraction (CRS type 2 and 4) is common especially in older females with hypertension and/or diabetes. It can be hypothesized that the incidence of this disease association is growing, while clinical trials enrolling these patients are still lacking. Differently from CRS in heart failure with reduced ejection fraction, the involvement of the kidney may be under-diagnosed in patients with heart failure and preserved ejection fraction and the optimal therapeutic strategy in this condition, though challenging, is far to be completely elucidated. Further studies are needed to assess the best therapeutic regimen in patients with renal dysfunction (and worsening) and heart failure and preserved ejection fraction.

"The people who bind themselves to systems are those who are unable to encompass the whole truth and try to catch it by the tail; a system is like the tail of truth, but the truth is like a lizard; it leaves its tail in your fingers and runs away knowing full well that it will grow a new one in a twinkling." (Ivan Turgenev to Leo Tolstoy)

\section{References}

[1] C. Ronco, M. Haapio, A. A. House, N. Anavekar, and R. Bellomo, "Cardiorenal syndrome," Journal of the American College of Cardiology, vol. 52, no. 19, pp. 1527-1539, 2008.

[2] C. Ronco, A. A. House, and M. Haapio, "Cardiorenal syndrome: refining the definition of a complex symbiosis gone wrong," Intensive Care Medicine, vol. 34, no. 5, pp. 957-962, 2008.

[3] C. Ronco, P. McCullough, S. D. Anker et al., "Cardio-renal syndromes: report from the consensus conference of the acute dialysis quality initiative," European Heart Journal, vol. 31, no. 6, pp. 703-711, 2010.

[4] G. L. Smith, J. H. Lichtman, M. B. Bracken et al., "Renal impairment and outcomes in heart failure. Systematic review and meta-analysis," Journal of the American College of Cardiology, vol. 47, no. 10, pp. 1987-1996, 2006.

[5] S. Kshatriya, H. Kozman, D. Siddiqui et al., "The cardiorenal syndrome in heart failure: an evolving paradigm," American Journal of the Medical Sciences, vol. 340, no. 1, pp. 33-37, 2010.

[6] J. S. Bock and S. S. Gottlieb, "Cardiorenal syndrome: new perspectives," Circulation, vol. 121, no. 23, pp. 2592-2600, 2010.

[7] H. L. Hillege, D. Nitsch, M. A. Pfeffer et al., "Renal function as a predictor of outcome in a broad spectrum of patients with heart failure," Circulation, vol. 113, no. 5, pp. 671-678, 2006.

[8] R. N. Foley, P. S. Parfrey, and M. J. Sarnak, "Epidemiology of cardiovascular disease in chronic renal disease," Journal of the American Society of Nephrology, vol. 9, no. 12, pp. S16-23, 1998.

[9] R. K. Wali, G. S. Wang, S. S. Gottlieb et al., "Effect of kidney transplantation on left ventricular systolic dysfunction and congestive heart failure in patients with end-stage renal disease," Journal of the American College of Cardiology, vol. 45, no. 7, pp. 1051-1060, 2005. 
[10] A. Levin, J. Singer, C. R. Thompson, H. Ross, and M. Lewis, "Prevalent left ventricular hypertrophy in the predialysis population: identifying opportunities for intervention," American Journal of Kidney Diseases, vol. 27, no. 3, pp. 347-354, 1996.

[11] P. S. Parfrey, R. N. Foley, J. D. Harnett, G. M. Kent, D. C. Murray, and P. E. Barre, "Outcome and risk factors for left ventricular disorders in chronic uraemia," Nephrology Dialysis Transplantation, vol. 11, no. 7, pp. 1277-1285, 1996.

[12] D. D. Correa de Sa, D. O. Hodge, J. P. Slusser et al., "Progression of preclinical diastolic dysfunction to the development of symptoms," Heart, vol. 96, no. 7, pp. 528-532, 2010.

[13] L. G. Bongartz, M. J. Cramer, P. A. Doevendans, J. A. Joles, and B. Braam, "The severe cardiorenal syndrome: 'Guyton revisited," European Heart Journal, vol. 26, no. 1, pp. 11-17, 2005.

[14] C. Ronco, A. A. House, and M. Haapio, "Cardiorenal and renocardiac syndromes: the need for a comprehensive classification and consensus," Nature Clinical Practice. Nephrology, vol. 4, no. 6, pp. 310-311, 2008.

[15] C. Ronco and A. Maisel, "Volume overload and cardiorenal syndromes," Congestive Heart Failure, vol. 16, supplement 1, pp. S1-S4, 2010.

[16] R. Tarquini, C. Lazzeri, L. Pala, C. M. Rotella, and G. F. Gensini, "The diabetic cardiomyopathy," Acta Diabetologica. In press.

[17] G. C. Fonarow, W. G. Stough, W. T. Abraham et al., "Characteristics, treatments, and outcomes of patients with preserved systolic function hospitalized for heart failure. A report from the OPTIMIZE-HF registry," Journal of the American College of Cardiology, vol. 50, no. 8, pp. 768-777, 2007.

[18] R. S. Bhatia, J. V. Tu, D. S. Lee et al., "Outcome of heart failure with preserved ejection fraction in a population-based study," New England Journal of Medicine, vol. 355, no. 3, pp. 260-269, 2006.

[19] K. McDonald, "Diastolic heart failure in the elderly: underlying mechanisms and clinical relevance," International Journal of Cardiology, vol. 125, no. 2, pp. 197-202, 2008.

[20] P. A. McCullough, A. K. Khandelwal, J. E. McKinnon et al., "Outcomes and prognostic factors of systolic as compared with diastolic heart failure in urban America," Congestive Heart Failure, vol. 11, no. 1, pp. 6-11, 2005.

[21] F. Tehrani, A. Phan, C. V. Chien, R. P. Morrissey, A. M. Rafique, and E. R. Schwarz, "Value of medical therapy in patients $>80$ years of age with heart failure and preserved ejection fraction," American Journal of Cardiology, vol. 103, no. 6, pp. 829-833, 2009.

[22] M. Fischer, A. Baessler, H. W. Hense et al., "Prevalence of left ventricular diastolic dysfunction in the community: results from a Doppler echocardiographic-based survey of a population sample," European Heart Journal, vol. 24, no. 4, pp. 320-328, 2003.

[23] G. C. Fonarow and ADHERE Scientific Advisory Committee, "The Acute Decompensated Heart Failure National Registry $\left(\mathrm{ADHERE}^{T M}\right)$ : opportunities to improve care of patients hospitalized with acute decompensated heart failure," Reviews in Cardiovascular Medicine, vol. 4, supplement 7, pp. S21-S30, 2003.

[24] W. J. Paulus and J. J. M. van Ballegoij, "Treatment of heart failure with normal ejection fraction. An inconvenient truth!," Journal of the American College of Cardiology, vol. 55, no. 6, pp. 526-537, 2010.

[25] T. E. Owan, D. O. Hodge, R. M. Herges, S. J. Jacobsen, V. L. Roger, and M. M. Redfield, "Trends in prevalence and outcome of heart failure with preserved ejection fraction,"
New England Journal of Medicine, vol. 355, no. 3, pp. 251-259, 2006.

[26] C. Tribouilloy, D. Rusinaru, H. Mahjoub et al., "Prognosis of heart failure with preserved ejection fraction: a 5 year prospective population-based study," European Heart Journal, vol. 29, no. 3, pp. 339-347, 2008.

[27] C. Tschöpe and D. Westermann, "Heart failure with normal ejection fraction: pathophysiology, diagnosis, and treatment," Herz, vol. 34, no. 2, pp. 89-96, 2009.

[28] M. Turakhia, S. Ali, N. B. Schiller et al., "Left ventricular mass predicts death and sudden death in patients with CAD regardless of ejection fraction: data from the Heart and Soul Study," Circulation, vol. 114, p. II-400, 2006.

[29] S. M. Al-Khatib, L. Shaw, C. M. O'Connor et al., "Sudden cardiac death in patients with diastolic heart failure," Circulation, vol. 114, p. II-404, 2006.

[30] G. P. Aurigemma, M. R. Zile, and W. H. Gaasch, "Contractile behavior of the left ventricle in diastolic heart failure: with emphasis on regional systolic function," Circulation, vol. 113, no. 2, pp. 296-304, 2006.

[31] M. R. Zile, C. F. Baicu, and W. H. Gaasch, "Diastolic heart failure-abnormalities in active relaxation and passive stiffness of the left ventricle," New England Journal of Medicine, vol. 350, no. 19, pp. 1953-2018, 2004.

[32] M. Kawaguchi, I. Hay, B. Fetics, and D. A. Kass, "Combined ventricular systolic and arterial stiffening in patients with heart failure and preserved ejection fraction: implications for systolic and diastolic reserve limitations," Circulation, vol. 107, no. 5, pp. 714-720, 2003.

[33] B. A. Borlaug, V. Melenovsky, S. D. Russell et al., "Impaired chronotropic and vasodilator reserves limit exercise capacity in patients with heart failure and a preserved ejection fraction," Circulation, vol. 114, no. 20, pp. 2138-2147, 2006.

[34] F. R. Winton, "The influence of venous pressure on the isolated mammalian kidney," Journal of Physiology, vol. 72, pp. 49-61, 1931.

[35] J. M. Doty, B. H. Saggi, H. J. Sugerman et al., "Effect of increased renal venous pressure on renal function," Journal of Trauma, vol. 47, no. 6, pp. 1000-1003, 1999.

[36] K. Damman, V. M. van Deursen, G. Navis, A. A. Voors, D. J. van Veldhuisen, and H. L. Hillege, "Increased central venous pressure is associated with impaired renal function and mortality in a broad spectrum of patients with cardiovascular disease," Journal of the American College of Cardiology, vol. 53, no. 7, pp. 582-588, 2009.

[37] H. Palomba, I. de Castro, A. L. C. Neto, S. Lage, and L. $\mathrm{Yu}$, "Acute kidney injury prediction following elective cardiac surgery: AKICS Score," Kidney International, vol. 72, no. 5, pp. 624-631, 2007.

[38] D. R. Meldrum, F. A. Moore, E. E. Moore, R. J. Franciose, A. Sauaia, and J. M. Burch, "Prospective characterization and selective management of the abdominal compartment syndrome," American Journal of Surgery, vol. 174, no. 6, pp. 667-673, 1997.

[39] A. Nohria, V. Hasselblad, A. Stebbins et al., "Cardiorenal interactions. Insights from the ESCAPE trial," Journal of the American College of Cardiology, vol. 51, no. 13, pp. 1268-1274, 2008.

[40] K. Damman, V. M. van Deursen, G. Navis, A. A. Voors, D. J. van Veldhuisen, and H. L. Hillege, "Increased central venous pressure is associated with impaired renal function and mortality in a broad spectrum of patients with cardiovascular disease," Journal of the American College of Cardiology, vol. 53, no. 7, pp. 582-588, 2009. 
[41] W. Mullens, Z. Abrahams, G. S. Francis et al., "Importance of venous congestion for worsening of renal function in advanced decompensated heart failure," Journal of the American College of Cardiology, vol. 53, no. 7, pp. 589-596, 2009.

[42] M. H. Drazner, J. E. Rame, L. W. Stevenson, and D. L. Dries, "Prognostic importance of elevated jugular venous pressure and a third heart sound in patients with heart failure," New England Journal of Medicine, vol. 345, no. 8, pp. 574-581, 2001.

[43] H. Krum, M. Schlaich, R. Whitbourn et al., "Catheter-based renal sympathetic denervation for resistant hypertension: a multicentre safety and proof-of-principle cohort study," Lancet, vol. 373, no. 9671, pp. 1275-1281, 2009.

[44] K. Dickstein, A. Cohen-Solal, G. Filippatos et al., "ESC Guidelines for the diagnosis and treatment of acute and chronic heart failure 2008. The Task Force for the Diagnosis and Treatment of Acute and Chronic Heart Failure 2008 of the European Society of Cardiology. Developed in collaboration with the Heart Failure Association of the ESC (HFA) and endorsed by the European Society of Intensive Care Medicine (ESICM)," European Journal of Heart Failure, vol. 10, no. 10, pp. 933-989, 2008.

[45] N. D. Vaziri, M. Dicus, N. D. Ho, L. Boroujerdi-Rad, and R. K. Sindhu, "Oxidative stress and dysregulation of superoxide dismutase and NADPH oxidase in renal insufficiency," Kidney International, vol. 63, no. 1, pp. 179-185, 2003.

[46] C. Heymes, J. K. Bendall, P. Ratajczak et al., "Increased myocardial NADPH oxidase activity in human heart failure," Journal of the American College of Cardiology, vol. 41, no. 12, pp. 2164-2171, 2003.

[47] K. K. Griendling, C. A. Minieri, J. D. Ollerenshaw, and R. W. Alexander, "Angiotensin II stimulates NADH and NADPH oxidase activity in cultured vascular smooth muscle cells," Circulation Research, vol. 74, no. 6, pp. 1141-1148, 1994.

[48] D. Villarreal, R. H. Freeman, and M. W. Brands, "DOCA administration and atrial natriuretic factor in dogs with chronic heart failure," American Journal of Physiology-Heart and Circulatory Physiology, vol. 257, no. 3, pp. H739-H745, 1989.

[49] J. Reinglas, H. Haddad, R. A. Davies, and L. Mielniczuk, "Cardiorenal syndrome and heart failure," Current Opinion in Cardiology, vol. 25, no. 2, pp. 141-147, 2010.

[50] A. B. Miller and I. L. Piña, "Understanding heart failure with preserved ejection fraction: clinical importance and future outlook," Congestive Heart Failure, vol. 15, no. 4, pp. 186-192, 2009.

[51] Y. Iwanaga and S. Miyazaki, "Heart failure, chronic kidney disease, and biomarkers - an integrated viewpoint-," Circulation Journal, vol. 74, no. 7, pp. 1274-1282, 2010.

[52] N. Hamdani and W. J. Paulus, "Treatment of heart failure with normal ejection fraction," Current Treatment Options in Cardiovascular Medicine, vol. 55, no. 6, pp. 526-537, 2010.

[53] M. R. Zile and D. L. Brutsaert, "New concepts in diastolic dysfunction and diastolic heart failure: part II. Causal mechanisms and treatment," Circulation, vol. 105, no. 12, pp. 15031508, 2002.

[54] N. Angomachalelis, A. I. Hourzamanis, S. Sideri, E. Serasli, and C. Vamvalis, "Improvement of left ventricular diastolic dysfunction in hypertensive patients 1 month after ACE inhibition therapy: evaluation by ultrasonic automated boundary detection," Heart and Vessels, vol. 11, no. 6, pp. 303-309, 1996.

[55] S. A. Hunt, D. W. Baker, M. H. Chin et al., "ACC/AHA guidelines for the evaluation and management of chronic heart failure in the adult: executive summary. A report of the American College of Cardiology/American Heart Association
Task Force on Practice Guidelines (Committee to Revise the 1995 Guidelines for the Evaluation and Management of Heart Failure)," Journal of the American College of Cardiology, vol. 38, no. 7, pp. 2101-2113, 2001.

[56] W. H. Gaasch and M. R. Zile, "Left ventricular diastolic dysfunction and diastolic heart failure," Annual Review of Medicine, vol. 55, pp. 373-394, 2004.

[57] S. Yusuf, M. A. Pfeffer, K. Swedberg et al., "Effects of candesartan in patients with chronic heart failure and preserved leftventricular ejection fraction: the CHARM-preserved trial," Lancet, vol. 362, no. 9386, pp. 777-781, 2003.

[58] V. Jorapur, G. A. Lamas, Z. P. Sadowski et al., "Renal impairment and heart failure with preserved ejection fraction early post-myocardial infarction," World Journal of Cardiology, vol. 2, no. 1, pp. 13-18, 2010.

[59] G. W. K. Yip, M. Wang, T. Wang et al., "The Hong Kong diastolic heart failure study: a randomised controlled trial of diuretics, irbesartan and ramipril on quality of life, exercise capacity, left ventricular global and regional function in heart failure with a normal ejection fraction," Heart, vol. 94, no. 5, pp. 573-580, 2008. 


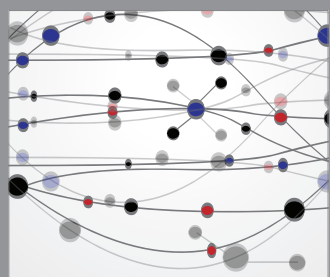

The Scientific World Journal
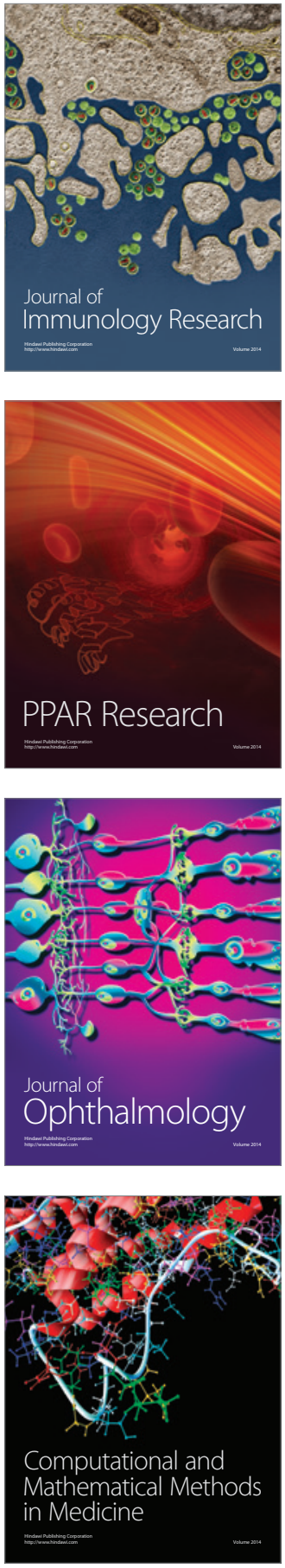

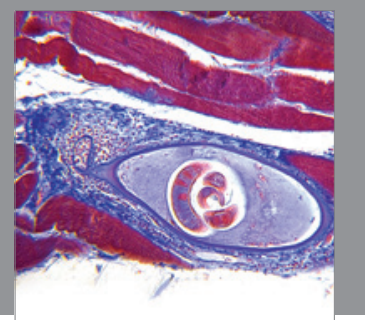

Gastroenterology

Research and Practice
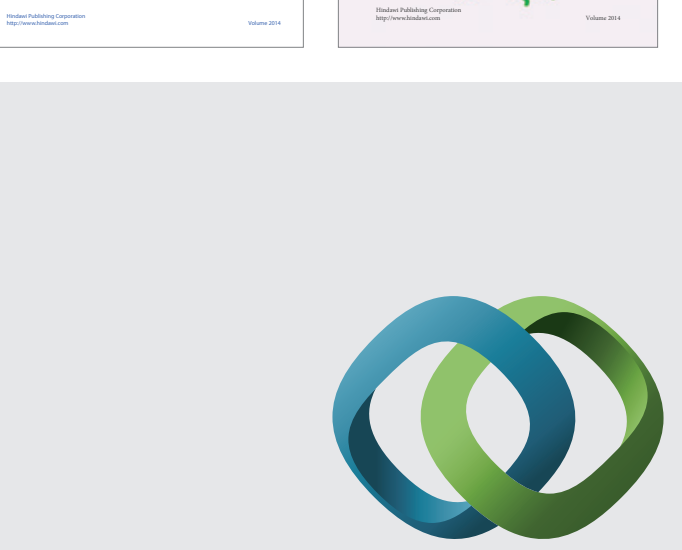

\section{Hindawi}

Submit your manuscripts at

http://www.hindawi.com
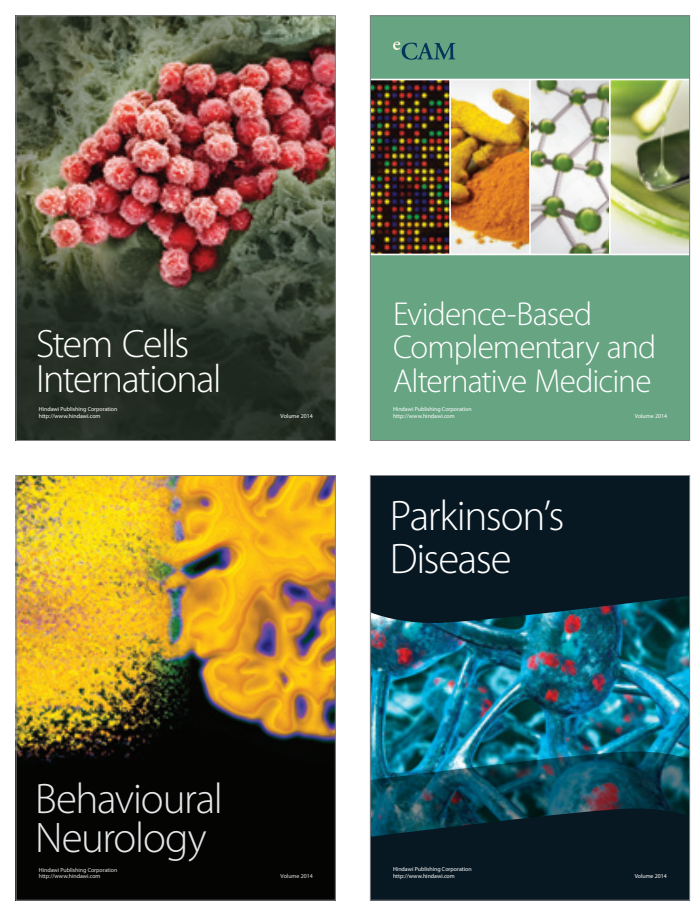

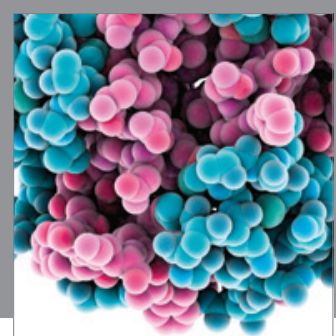

Journal of
Diabetes Research

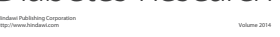

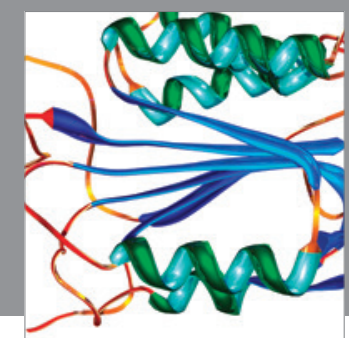

Disease Markers
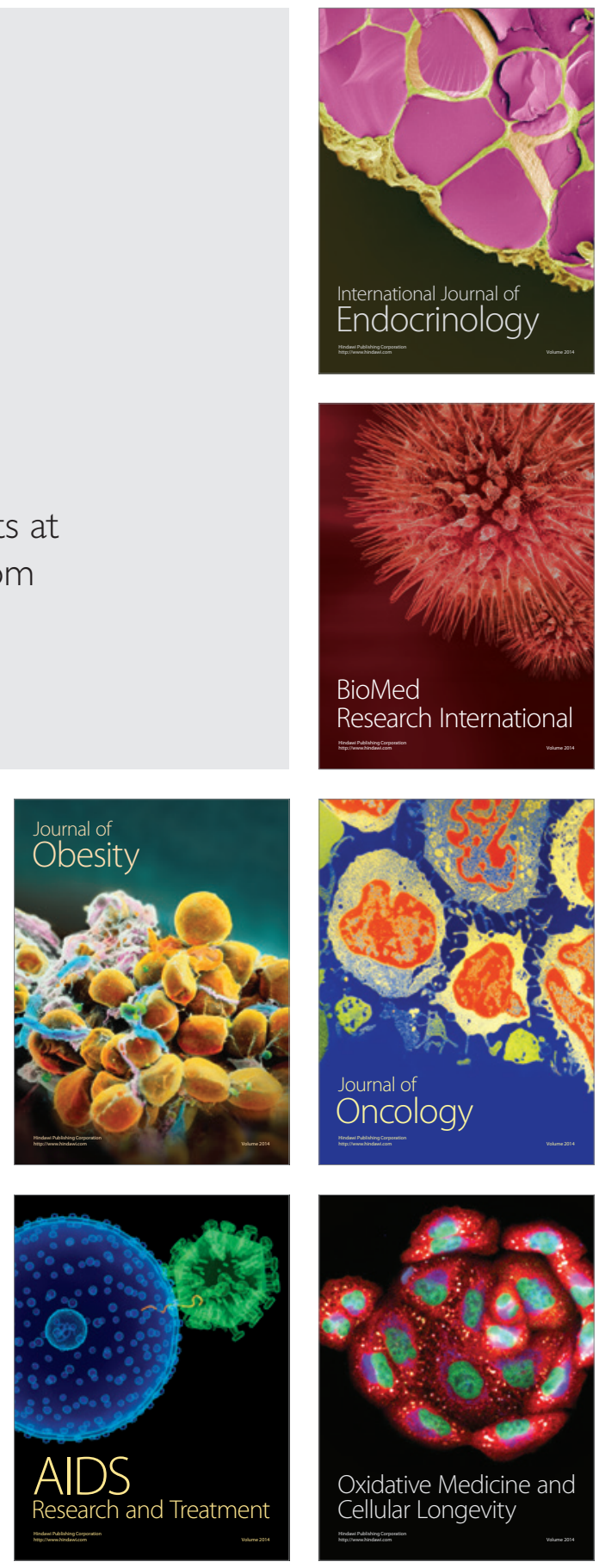